\title{
Dictynna
}

Dictynna

Revue de poétique latine

16 | 2019

Varia

\section{En attendant Achille (Stace, Achilléide, I, 467-513) : enjeux dramatiques, éthiques et politiques d'une scène « de transition »}

\section{François Ripoll}

\section{(2) OpenEdition}

Journals

Édition électronique

URL : http://journals.openedition.org/dictynna/1826

DOI : $10.4000 /$ dictynna. 1826

ISSN : 1765-3142

Référence électronique

François Ripoll, «En attendant Achille (Stace, Achilléide, I, 467-513):

enjeux dramatiques, éthiques et politiques d'une scène « de transition » », Dictynna [En ligne], 16 |

2019, mis en ligne le 25 novembre 2019, consulté le 11 septembre 2020. URL : http://

journals.openedition.org/dictynna/1826 ; DOI : https://doi.org/10.4000/dictynna.1826

Ce document a été généré automatiquement le 11 septembre 2020.

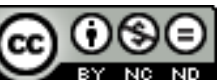

Les contenus des la revue Dictynna sont mis à disposition selon les termes de la Licence Creative Commons Attribution - Pas d'Utilisation Commerciale - Pas de Modification 4.0 International. 


\title{
En attendant Achille (Stace, Achilléide, I, 467-513) : enjeux dramatiques, éthiques et politiques d'une scène « de transition »
}

\author{
François Ripoll
}

1 En dépit de la floraison récente d'études sur l'Achilléide de Stace, la longue section centrale (397-558) consacrée au moment où les Grecs, rassemblés à Aulis, vont décider d'envoyer une délégation pour aller chercher le jeune héros, a peu retenu l'attention des critiques ; ceux-ci s'intéressent davantage, en effet, aux épisodes d'allure élégiaque et ovidienne consacrés à sa liaison avec Deidamie, au thème de son travestissement et de son ambiguïté sexuelle, ou à la scène dramatique de sa découverte parmi les filles de Lycomède. Cela vient sans doute de ce que l'ambiance néo-homérique de l'épisode d'Aulis tranche avec la couleur alexandrine certes prédominante, mais non exclusive, dans le reste du poème, et qui suscite davantage la sympathie de la critique. Encore faut-il introduire une nuance : la prophétie de Calchas (514-537) a été plus souvent prise en compte que les préparatifs guerriers de l'armée argienne (397-466), et surtout, que la section des v. 467-513, qui fait office de transition entre le catalogue et la scène de divination ${ }^{1}$.

2 Rappelons la composition de ce passage, que nous nous proposons d'examiner de près. La première partie (467-490) montre l'armée grecque réclamant Achille: celle-ci commence par passer en revue les chefs Achéens éclipsés par le jeune héros dans le cœur des soldats (467-475); suit un discours semi-direct reproduisant les propos élogieux des soldats sur Achille (476-482) : son éducation rude (476-478), ses origines divines (479), son immunisation par le Styx (480-481); la section se termine sur une comparaison de l'armée suspendue à l'arrivée d'Achille aux Olympiens dans l'attente de l'intervention de Jupiter contre les Géants (483-490). La seconde partie (491-513) met en 
scène Protésilas interpellant Calchas. A l'évocation de son attitude agressive (491-495) succède un discours direct (496-513) : reproches de lenteur à Calchas (496-498); passage en revue sur un ton amer des chefs éclipsés par Achille (499-504); exhortation pressante à Calchas à révéler la cachette d'Achille (504-509); rappel des privilèges du devin et promesse d'honneurs (510-513).

Texte et traduction ${ }^{2}$.

Sed quanquam et gemini pariter sua bella capessant

Atridae famamque auida uirtute paternam

Tydides Sthenelusque premat, nec cogitet annos

Antilochus septemque Aiax umbone coruscet armenti reges atque aequum moenibus orbem, consiliisque armisque uigil contendat Vlixes, omnis in absentem belli manus ardet Achillem, nomen Achillis amant et in Hectora solus Achilles poscitur, illum unum Teucris Priamoque loquuntur fatalem : «Quis enim Haemoniis sub uallibus alter creuerit effossa reptans niue ? Cuius adortus cruda rudimenta et teneros formauerit annos Centaurus ? Patrii propior cui linea Caeli, quemue alium Stygios tulerit secreta per amnes Nereis et pulchros ferro praestruxerit artus?» Haec Graiae castris iterant traduntque cohortes. Cedit turba ducum, uincique haud maesta fatetur. Sic cum pallentes Phlegraea in castra coirent caelicolae iamque Odrysiam Gradiuus in hastam surgeret et Libycos Tritonia tolleret angues ingentemque manu curuaret Delius arcum, stabat anhela metu solum Natura Tonantem respiciens, quando ille hiemes tonitrusque uocaret nubibus, igniferam quot fulmina posceret Aetnen. Atque ibi dum mixta uallati plebe suorum et maris et belli consultant tempora reges, increpitans magno uatem Calchanta tumultu Protesilaus agit - namque huic bellare cupido praecipua et primae iam tunc data gloria mortis - ; "O nimium Phoebi tripodumque oblite tuorum, Thestoride, quando ora deo possessa mouebis iustius, aut quianam Parcarum occulta recludes? Cernis ut ignotum cuncti stupeantque fremantque Aeaciden? Sordent uulgo Calydonius heros et magno genitus Telamone Aiaxque secundus, nos quoque ; sed Mauors et Troia arrepta probabunt. Illum neglectis - pudet heu ! - ductoribus omnes belligerum ceu numen amant. Dic ocius, aut cur serta comis et multus honos? Quibus abditus oris? Quaue iubes tellure peti? Nam fama nec antris Chironis, patria nec degere Peleos aula. Heia, inrumpe deos et fata latentia uexa, laurigerosque ignes, si quando, auidissimus hauri ! Arma horrenda tibi saeuosque remisimus enses, numquam has inbelles galea uiolabere uittas, sed felix numeroque ducum praestantior omni, si magnum Danais pro te dependis Achillem. » 
Mais si les deux Atrides, d'un même élan, se jettent dans leur guerre, si le fils de Tydée ainsi que Sthénélus cherchent à éclipser la gloire paternelle, si Antiloque oublie le nombre de ses années, si Ajax fait étinceler, sur son bouclier, sept rois du troupeau et un orbe aussi vaste que des remparts, si Ulysse, toujours en éveil, est fort de ses conseils et de ses armes, tout entière pour un absent brûle la troupe guerrière : Achille. C'est le nom d'Achille qu'ils chérissent, et c'est, contre Hector, Achille seul que l'on réclame; lui seul, dit-on, sera fatal aux Troyens et à Priam. Quel autre, au creux des vallées d'Hémonie, a grandi en rampant dans la neige creusée ? Qui d'autre a reçu une rude éducation et a été formé, en ses tendres années, par les leçons d'un Centaure ? De qui le lignage touche-t-il de plus près au Ciel paternel ? Qui d'autre fut porté en secret, dans les ondes stygiennes, par une Néréide, qui protégea du fer son beau corps? Voilà ce que répètent et propagent dans le camp les cohortes grecques. La foule des chefs s'incline, et sans rancœur s'avoue vaincue. Ainsi lorsqu'aux camps phlégréens s'assemblaient, pâlissants, les habitants du Ciel, que déjà, appuyé sur sa lance odrysienne, Gradivus se dressait, que la Tritonienne brandissait ses serpents libyens, que le bras du Délien courbait son arc immense, alors, haletante d'effroi, la Nature, figée, regardait le seul Tonnant : quand appellerait-il des nuées orages et tonnerre? Combien de foudres demanderait-il à la forge de l'Etna?

Et tandis qu'entourés de la foule mêlée de leurs hommes, les rois discutent du meilleur temps pour la mer et pour la guerre, Protésilas invective à grand bruit le devin Calchas - domine en lui le désir de la guerre, et déjà lui est donnée la gloire de mourir le premier - : «O Thestoride trop oublieux de Phébus et de tes trépieds, quand ouvriras-tu ta bouche, possédée du dieu, mieux à propos, et quel motif te poussera enfin à révéler les secrets des Parques? Vois-tu comme tous, sans le connaître, acclament, fascinés, l'Eacide? La foule n'a que mépris pour le héros de Calydon, pour le fils du grand Télamon, pour le second Ajax, et pour moi aussi ; mais Mars et la prise de Troie prouveront notre valeur. C'est lui que tous, dédaignant (honte à nous!) les grands chefs, chérissent comme une divinité guerrière. Parle au plus vite; ou à quoi bon cette couronne sur ta tête et tous les honneurs dont tu jouis? Sur quels rivages se cache-t-il, ou sur quelle terre nous enjoins-tu de le chercher? Car il ne vit, dit-on, ni dans l'antre de Chiron, ni à la cour de son père Pélée. Allons, fais irruption chez les dieux, harcèle les destins cachés, hume avec plus d'avidité que jamais les feux du laurier! Nous t'avons exempté des armes redoutables et des cruelles épées, jamais casque ne profanera ces bandelettes pacifiques, mais tu auras le bonheur d'être placé au-dessus de tous les chefs réunis si tu donnes aux Danaens, pour te remplacer, le grand Achille».

3 Ce passage négligé, dans la mesure précisément où il brasse des thématiques différentes de celles de l'intrigue centrale, permet d'apporter un éclairage complémentaire sur le projet poétique de l'Achilléide et de nuancer l'image un peu monolithique que l'on en donne parfois. J'envisagerai trois axes problématiques :

- Le thème de l'attente du héros et l'orchestration de la tension dramatique.

- L'introduction et la caractérisation par le discours (éthopée) d'une figure secondaire : Protésilas.

- Les enjeux socio-politiques qui sous-tendent la mise en scène de la collectivité guerrière dans ses diverses composantes.

\section{L'attente du héros.}

L'idée de développer ce thème doit dériver indirectement du début du chant IX de l' Iliade, quand les chefs grecs, mis en difficulté, se rendent compte à quel point Achille leur manque (89-172). Stace y a puisé l'idée fondamentale selon laquelle l'entrée en jeu d'Achille est l'objet d'une vive attente collective, ce qui lui permettait de mettre en 
valeur la figure de son héros encore enfant en faisant entrevoir proleptiquement à travers lui le futur "super-guerrier » iliadique à l'intervention duquel tout le monde serait un jour suspendu. Ce type de jeu allusif par « répétition anticipée » d'une scène homérique est un procédé récurrent de l'Achilléide, qui, en tant que prequel de l'Iliade et de l'odyssée, cherche à offrir à son lecteur le plaisir de la reconnaissance proleptique ${ }^{3}$. Toutefois cette influence d'Iliade IX reste à un niveau très général et n'a laissé aucune trace intertextuelle précise dans le texte de Stace. En fait, ce dernier a conçu l'idée, d'une part, de développer cette idée d'attente sous la forme d'une synkrisis antithétique entre Achille et les autres chefs grecs infériorisés par rapport à lui, et d'autre part, de subjectiviser cette synkrisis en la plaçant dans la bouche de la troupe. Or il me semble que ce double parti pris, qui dramatise puissamment la scène de l'attente, a été suggéré au poète de l'Achilléide par un de ses prédécesseurs proches : Valérius Flaccus, chez qui l'on trouve précisément une scène dans laquelle l'attente d'un héros majeur est source de tension dans une scène dominée par les discours 4 . Il s'agit du débat des Argonautes après la disparition d'Hercule ${ }^{5}$ (Arg., III, 598-714). Plusieurs éléments semblent indiquer que Stace avait le précédent valérien à l'esprit. D'une part, le mouvement initial par quanquam énumérant les chefs achéens supplantés par Achille (467) rappelle les v. 599-600 de Valérius, où la concessive oppose Hylas, certes apprécié de tous, à Hercule, bien plus regretté que lui (quamquam omnibus aeque/grata rudimenta). On trouve dans ce même mouvement l'expression Herculeo sub nomine pendent (600), auquel répond ici : nomen Achillis amant (v. 474), avec dans les deux cas, la relance par un illum antéposé voisinant avec le verbe poscere ${ }^{6}$ (Ach. $475:$... poscitur, illum... et Arg. 601: illum... reposcere). La junctura valérienne grata rudimenta (600) a en outre un écho dans le cruda rudimenta de Stace (478), à la même place dans le vers. Plus précisément, le discours de Méléagre chez Valérius a laissé une trace assez nette dans celui de Protésilas: le moment ou le locuteur déroule, sur un ton plus ou moins dépité, un liste de chefs jugés inférieurs au héros principal, en finissant par lui-même, et en promettant des exploits à venir qui prouveront sa propre valeur (500-502: cf. Arg., III, 667-727). Aussi n'est-ce peut-être pas par hasard que Protésilas désigne Diomède comme Calydonius heros (500), une épithète couramment associée à... Méléagre (cf. VF I, 646: Magnanimus Calydone satus ${ }^{8}$ ). Nous verrons que l'intertexte valérien a aussi été convoqué par Stace pour un motif connexe: le conflit latent que l'attente du héros met à jour au sein de la collectivité (sous une forme nettement atténuée par rapport à Valérius).

Sur le plan de la cohésion du récit, Stace était toutefois confronté à une petite difficulté. Le choix de développer le motif de l'attente du héros majeur en prélude à la scène de vaticination impliquait que les Achéens aient déjà de fortes raisons de souhaiter l'entrée en jeu d'Achille préalablement à l'intervention de Calchas. Or d'après la tradition légendaire issue des Chants Cypriens, représentée notamment par les Skyrioi d'Euripide, et reprise par le pseudo-Apollodore (III, 13, 8), c'est une prophétie, parfois attribuée à Calchas, qui avait révélé aux Achéens qu'Achille était indispensable à l'expédition contre Troie (la même prophétie devait, dans les Skyrioi, indiquer le lieu de cachette d'Achille甲). Le choix structurel opéré par Stace lui impose de redistribuer la matière de la prophétie de Calchas : les Achéens savent déjà qu'ils ont besoin d'Achille (pour des raisons que je vais préciser dans un instant), et la vaticination de Calchas (qui, du coup, n'est plus à proprement parler une prophétie) leur donnera seulement des indices sur l'endroit où le chercher. On voit donc que chez Stace, l'effet affectif et dramatique dans la mise en scène de l'attente du héros était une suggestion que le 
poète tenait à developper en elle-même, au prix de quelques aménagements de la tradition légendaire.

6 C'est aux v. 475-81 que sont exposées les raisons de l'espérance qu'Achille suscite chez les Grecs. La première idée énoncée est celle d'une prédestination d'Achille à la victoire sur Hector et, consécutivement, au renversement de la puissance troyenne. Cette croyance découlait originellement de l'oracle de Calchas ; mais comme Stace, on l'a vu, a décidé de postposer la scène oraculaire, il ne peut plus faire référence à un oracle antérieur. Cette prédestination se trouve dès lors ramenée à un "on-dit» (loquuntur) dont l'origine n'est pas précisée : c'est en fait la tradition littéraire sur Achille tombeur d'Hector et fossoyeur prédestiné de Troie, tradition supposée anachroniquement connue des personnages sous forme d'une vague rumeur, qui est ici le support implicite de la connaissance, comme cela arrive souvent dans la poésie néo-alexandrine ${ }^{10}$. Mais cette confiance envers celui qui n'est encore qu'un enfant et n'a accompli aucun exploit, si elle n'est pas fondée sur une prophétie en bonne et due forme, doit tout de même être étayée au niveau du récit par des éléments plus objectifs qu'une simple rumeur. C'est le rôle de la séquence en discours indirect libre des v. 476-81, qui équivaut à une laudatio d'Achille, sous la forme d'une quadruple série de questions oratoires (anaphore du pronom interrogatif avec polyptote), fondées sur une base comparative avec variations (quis alter... quem alium), de façon à donner à la primauté d'Achille l'allure d'une évidence censée s'imposer à tous. L'énumération se déploie en trois temps, recoupant tout ce que les Achéens sont supposés savoir du Péléide à ce stade : son éducation (475-79)/ son origine divine (479)/ son immunisation par le Styx (480-82), dans une progression organisée du plus naturel au plus surnaturel. L'éducation d'Achille sous la rude férule de Chiron se fonde sur une tradition bien connue développée notamment dans la troisième Néméenne de Pindare ${ }^{11}$. Ici, Stace se limite à deux indications suggestives d'une éducation "à la dure »: une hypotypose concrète (l'enfant rampant dans la neige) et un condensé général et abstrait (le rôle formateur de Chiron). Le premier motif s'inspire directement de l'éducation de l'Arcadien Parthénopée dans la Thébaïde, IX, 79712 (l'enfant rampant sur les fleuves gelés), qui elle-même s'inspirait de l'exposé de Numanus Rémulus sur la rude éducation de la jeunesse italique chez Virgile (Aen. IX, 603-604), prototype du topos épique de l'éducation rigoureuse des peuples primitifs ${ }^{13}$. La variation par substitution de la neige (dans laquelle l'enfant creuse une sorte de sillon en rampant dessus : effosa reptans niue) aux fleuves glacés s'accorde au cadre Hémonien, lieu de l'enfance d'Achille ${ }^{14}$, dont le relief montagneux et le climat froid suscitent autour du jeune bébé l'image d'un locus horridus sauvage et oppressant (Haemoniis sub uallibus ${ }^{15}$ ). Ce contraste entre la fragilité apparente de l'enfant et la rudesse de sa formation se retrouve dans l'évocation du rôle de Chiron $^{16}$ (antithèse cruda ${ }^{17} /$ teneros) ; un Chiron dont l'importance est soulignée par la structure binaire avec antéposition du premier verbe (adortus sous-ent. est), et le rejet du nom qui pointe la nature insolite du magister (Centaurus). Le deuxième motif de la laudatio est le lignage divin du héros, « proche du ciel paternel » (patrii propior... linea ${ }^{18}$ caeli), ce qui renvoie soit au motif de la quasi-filiation jovienne d'Achille, soit à son trisaïeul paternel Ouranos ${ }^{19}$, soit les deux à la fois. Après cette double série de considérations (qui recoupe en gros les catégories de l'acquis et de l'inné), la laudatio s'achève sur l'allusion à l'épisode de l'immersion dans le Styx, qui est l'atout le plus spécifique d'Achille. Cette version de l'immunisation d'Achille, d'origine probablement hellénistique, était encore peu répandue à la fin du $1^{\mathrm{er}}$ siècle, et Stace a manifestement contibué à la populariser, à défaut de l'avoir inventée ${ }^{20}$. Elle est en tout cas mise en 
relief ici par la métaphore ferro ${ }^{21}$ praestruxerit, qui évoque l'image d'une cuirasse ${ }^{22}$, et l'antithèse (soulignée par l'hyperbate) entre la beauté délicate d'Achille (pulchros... $\operatorname{artus}^{23}$ ) et sa vocation guerrière (ferro). Notons l'ironie (involontaire) dans l'allusion finale au rôle protecteur de Thétis: les Grecs qui se félicitent de cette action préservatrice de la Néréide sur son fils ne savent pas encore qu'elle est en train de pousser jusqu'au bout cette logique en cachant Achille à Scyros, au détriment cette fois de leur intérêt (l'adverbe secreto ${ }^{24}$ appuie l'analogie d'ambiance entre la ruse de Scyros et l'immersion dans le Styx: Thétis agit toujours subrepticement). Cette laudatio des virtualités martiales d'Achille s'achève donc sur une touche involontairement ambiguë. L'intérêt de cette séquence est aussi de délivrer un certain nombre de renseignements sur la petite enfance d'Achille que Stace, pour ne pas commencer son récit $a b$ ou ${ }^{25}$, n'a pas inclus dans sa narration initiale. Elle anticipe toutefois le récit plus complet de son enfance que fera Achille au chant II, 86-167, où certains des motifs présents ici seront repris et développés (rôle de Chiron notamment). Parallèlement, les origines de la guerre de Troie qui sont exposées ici seront reprises dans le discours d'Ulysse (II, 49-85). C'est ainsi un double éclairage compémentaire qui est porté obliquement sur les origines à la fois du conflit guerrier et du héros principal ${ }^{26}$.

7 Après les deux vers conclusifs de ce passage de discours (482-83), la séquence s'achève sur le «point d'orgue » d'une comparaison amplifiante, qui dramatise cette suggestion d'une attente suspendue à l'entrée en scène d'un héros majeur. L'armée achéenne tendue dans l'expectative d'Achille est comparée aux Olympiens attendant la réaction de Jupiter lors de la Gigantomachie. Cette comparaison est un bel exemple de combinaison de sources et de jeu de correspondances multiples comme les aime Stace et l'on peut tenter d'en reconstituer la genèse imaginative ${ }^{27}$. Le contexte de préparatifs guerriers en vue d'un affrontement d'ampleur planétaire a amené à l'esprit de Stace le souvenir d'une comparaison gigantomachique de Lucain dans un contexte analogue (VII, 145-50). Il lui a emprunté l'idée d'une énumération de dieux olympiens avec pour chacun son attribut typique ${ }^{28}$ (ce qui fait écho au petit catalogue des chefs achéens des v. 467-72) culminant dans l'évocation de Jupiter (auquel correspond Achille dans le comparée ${ }^{29}$, ce qui rappelle sa "quasi-filiation jovienne »). En outre, afin de dramatiser l'ambiance de cette comparaison, Stace s'est souvenu d'un passage de Valérius Flaccus (II, 16-20) lié dans sa mémoire à celui de Lucain par le motif gigantomachique commun (avec aussi la mention de Pallène) : il s'agit du motif de la peur collective des dieux ${ }^{30}$ (cf. Arg. II, 16: metus ecce deum ${ }^{31}$ ). L'introduction de ce motif de la peur, redoublé par l'apparition de Natura, sert ici à accentuer l'ambiance d'attente fébrile en prélude à l'entrée en scène du " numéro un » : Jupiter/ Achille ${ }^{32}$. Pour exprimer cette idée, Stace a songé à un autre passage de Valérius Flaccus qu'a sans doute appelé à son esprit l'allusion de Lucain à l'action météorologique de Jupiter dans Phars. VII, 151: la comparaison des Argonautes attaquant les Dolions en rangs serrés à un nuage d'orage dont on ne sait où il va frapper la terre (VF III, 90-94). Stace lui a emprunté le motif du spectateur angoissé dans l'attente de la foudre (en remplaçant les humains par la Nature pour amplifier la résonance cosmique de la scène) ainsi que la structure syntaxique d'ensemble (metu à l'ablatif commandant une double interrogative indirecte paratactique). Le motif de la peur est certes sans correspondant direct dans le comparé (c'est au contraire dans une ambiance d'espérance fervente que les Grecs attendent la venue d'Achille), mais l'analogie se situe sur un plan plus général: celui d'une vive intensité émotionnelle liée à une expectative collective. Au final, cette ample comparaison contribue à prolonger l'attente tout en la dramatisant. On est assez 
proche ici des effets propres à l'esthétique du Sublime ${ }^{33}$ : gradation affective culminant sur une touche de tension dramatique exacerbée, mise en jeu de phénomènes météorologiques impressionnants (foudre, tonnerre), schèmes de verticalité (surgeret, tolleret), image de grandeur (ingentem), résonance cosmique de l'événement, magnitudo animi d'Achille à l'arrière-plan... Cette recherche des effets sublimes, aux antipodes de l'ambiance esthétique dominante de l'Achilléide, crée un contraste avec les événements de Scyros, ce qui met en valeur une tension interne au caractère du héros principal. Ce même Achille que nous voyons sous les traits charmants d'un puer immature et efféminé de type alexandrin au niveau de la narration principale est en même temps le héros prédestiné d'un univers épique grandiose et tonitruant que ce passage nous fait entrevoir par le biais de la comparaison ${ }^{34}$. Stace pointe ainsi la dualité de son héros et l'ambivalence du projet épique de l'Achilléide, qui rend hommage à l'épopée « pathétique ${ }^{35}$ » d'ascendance iliadique tout en circonscrivant son espace quantitatif.

\section{La figure de Protésilas.}

L'entrée en scène de ce personnage à ce stade du récit, sans parallèle connu dans les sources, peut apparaître comme une rallonge superflue, qui ne fait que retarder un peu plus la révélation de Calchas: celui-ci aurait très bien pu entrer en scène immédiatement après le v. 490, sans avoir besoin de se faire aiguillonner par Protésilas pour se décider à entrer en transe. Mais Stace tenait à cette scène, qui lui permet de renforcer l'ambiance de frénésie guerrière dans laquelle baigne tout l'épisode d'Aulis en mettant en scène un caractère individuel synecdotique de l'état d'esprit général. Là encore, l'intéressant est de reconstituer la démarche imaginative du poète. Ayant conçu dès l'origine le projet de faire intervenir une vaticination de Calchas, Stace a immédiatement pensé à la scène de la fausse prophétie du même Calchas dans le récit mensonger de Sinon au chant II de l'Enéide (122-129) : de là vient l'idée d'introduire un chef achéen qui brutalise le devin réticent à délivrer son savoir ${ }^{36}$ (en remplaçant Ulysse par Protésilas pour des raisons que nous allons préciser). Simultanément, le poète a dû penser aussi à la scène de sa Thébaïde où Capanée invective, pour des raisons analogues, le devin Amphiaraos ${ }^{37}$ (Theb., III, 598-670), et en a tiré l'idée de donner une seconde fois la parole en discours direct à une figure de guerrier impatient et peu respectueux de la fonction religieuse. Cette scène d'invectives d'un guerrier à un devin se rattache, via la Thébaïde, au thème plus général du guerrier impie outrageant le devin, dont les antécédents, en amont du Capanée statien, sont notamment le Tydée d'Eschyle (Sept., 380-84) et l'Idas d'Apollonios de Rhodes ${ }^{38}$ (Arg., I, 485-491). On retrouve un peu de cette opposition ancienne du prêtre et du guerrier dans les v. 510-511, où Protésilas souligne le caractère imbellis du devin (nous y reviendrons). Mais l'Achilléide présente une version très atténuée de ce topos, dans la mesure où Protésilas, s'il manifeste un tempérament emporté et agressif, n'est pas vraiment un impie, et ne met pas réellement en doute la compétence de Calchas. Par ailleurs, pour étoffer le discours exhortatif du guerrier, Stace a songé à nouveau au discours de Méléagre chez Valérius Flaccus, en reprenant (sous une forme certes très atténuée) l'idée d'une jalousie latente du locuteur envers le héros que tous appellent (là, Hercule; ici, Achille); de là vient le mouvement des v. 500-504, ou le locuteur passe en revue les autres chefs mésestimés en finissant par lui-même, avec la promesse d'exploits à venir : cf. VF III, 667-72. Quant au choix du héros chargé ici d'invectiver le uates, Stace a eu une idée que l'on peut qualifier d'originale: celle de confier ce rôle à Protésilas. Rien dans la tradition 
mythique relative à ce personnage ne le mettait en conflit direct avec Calchas ${ }^{39}$, mais le choix s'explique à la fois par des considérations éthiques (ce guerrier traditionnellement belliqueux et impatient était ici plus convaincant que le prudent Ulysse dans le rôle du rudoyeur de devin), et par l'exploitation de l'ironie tragique : ce héros si pressé d'en découdre sera le premier à succomber devant Troie, sans pouvoir réaliser sa promesse du v. 502. Or l'ironie proleptique (le plus souvent souriante, mais tragique ici) est un procédé typique d'un prequel que l'on retrouve ailleurs dans l' Achilléide $^{40}$. Protésilas est donc représentatif d'une attitude profondément humaine et courante vis-à-vis de la divination, faite de fébrilité anxieuse et d'impatience vaine, en homme qui se précipite tête baissée vers un destin bien éloigné de ses propres attentes.

Procédons maintenant à l'analyse suivie du discours.

L'exorde (496-98) contamine Theb. VII, 547 (heu nimium... oblite tuorum) pour le ton de reproche et Theb. VIII, 117 (tripodum iam non memimisse meorum) pour le motif du devin oubliant sa spécialitéti (avec ici, effet de surenchère par l'ajout de Phoebi); la contamination vise à outrager le devin en mettant directement en cause sa compétence professionnelle sur un ton mordant. La double interrogative qui suit induit, en même temps qu'une progression diachronique anticipant sur l'énonciation de la prophétie, un effet de crescendo dans l'impatience et l'exaspération ${ }^{42}$.

Protésilas aborde ensuite ce qui correspondrait, dans un discours persuasif, à la narratio (499-504). Cela prend la forme d'une hypotypose de l'armée suspendue dans l'attente d'Achille qui reprend avec un effet d'écho et de variation les v. 467-75. Le redoublement verbal avec polysyndète (stupeantque fremantque) souligne l'enthousiasme collectif. L'oxymore qu'il forme avec ignotum, mis en valeur par l'hyperbate, suggère le paradoxe de cette popularité d'un point de vue "aristocratique» (qui est celui de Protésilas). D'où la métaphore hyperbolique sordent qui traduit une pointe de dépit, relancée par l'exclamation en incise pudet heu et reprise in fine par l'ablatif absolu neglectis ductoribus (503). L'énumération de chefs grecs que déroule Protésilas fait écho à celle des v. 468-72 avec quelques variations (ici : Diomède, les deux Ajax, Protésilas lui-même, c'est-à-dire pour l'essentiel des figures plus spécialisées dans la prouesse guerrière que précédemment), et un ton emphatique (périphrases) censé renforcer le contraste entre la gloire de ces grands chefs et le mépris dont ils sont l'objet. La déclaration pleine de fiducia du v. 502 fait retentir, par le biais du zeugma (Mauors et Troia arrepta ${ }^{43}$ ), les prétentions épiques du personnage frappées par avance d'ironie tragique. Le mouvement récapitulatif des v. 503-504 constitue le climax de la séquence en esquissant l'idée d'une quasi divinisation d'Achille par la troupe, en un mouvement inspiré de Lucain, VI, 253-54 $4^{44}$.

11 Vient ensuite la confirmatio (504-509). Le retour à la structure exhortative (impératif lapidaire : dic ocius) rappelle l'impatience du locuteur, prolongée via le mouvement alternatif (aut cur...) d'une question oratoire qui esquisse une feinte mise en doute de la compétence du devin (appuyée sur le zeugma et rendue plus pressante par l'ellipse verbale). Cela rappelle l'invective du v. 496, et relance le questionnement avec une insistance accrue. Dans les v. 505-506, la question se fait plus précise; à travers la double interrogative directe avec redondance (quibus... oris/ quaue... tellure), c'est bien le lieu où se trouve Achille plus que son destin qui est en question, comme l'appuie la confirmation par l'état de la rumeur aux v. 506-507 (fama: cf. loquuntur) : cette précision en incise fait gagner du temps au récit en présentant comme révolue l'étape de l'enquête à Phthie et sur le Pélion, au prix d'une ellipse narrative, pour tourner la 
quête à venir vers les rivages lointaines. La mise hors de cause des deux figures paternelles (Chiron et Pélée ${ }^{45}$ ) laisse pressentir en filigrane le caractère féminin et maternel de la dissimulation d'Achille, que la prophétie de Calchas confirmera. Ce discours prépare donc bien la voie à la vaticination de ce dernier, en circonscrivant par avance le champ de la révélation attendue. Après cette courte digression explicative, la structure exhortative reprend avec vigueur dans un tricolon ascendant (508-509) avec progression du général et particulier et de l'abstrait au concret, pour dépeindre la pratique de la mantique à laquelle Calchas est prié de s'adonner activement. La première partie du tricolon (irrumpe deos) est une brachylogie inspirée de Theb. III, 634 (superumque irrumpere coetus) et 549 (irrupisse uolantum concilia), dans le même contexte ; l'idée est celle d'un "coup de force» du devin en pleine assemblée divine comme métaphore violente de la pratique divinatoire. La seconde exhortation prolonge avec variation (substitution de fata à deos) l'isotopie guerrière sous-jacente de l'image précédente (irrumpere, uexare) : au yeux de ce va-t-en-guerre invétéré qu'est Protésilas, l'activité divinatoire apparaît comme une opération militaire : la " prise d'assaut » de la " place forte» divine. La troisième exhortation revient à un registre plus religieux, mais avec une vigoureuse brachylogie (ignes... hauri au sens de "absorbe les vapeurs sacrées ${ }^{46} »$ ), appuyée d'une hyperbole (auidissimus) et d'une junctura solennelle et apparemment originale (laurigeros ${ }^{47}$ ignes). Malgré la vigueur des images, le ton se fait moins agressif en cette fin de discours : Protésilas ne feint plus de mettre en cause la compétence de Calchas, mais l'invite à donner la pleine mesure de celle-ci, et affiche même, par le biais de ses métaphores, une confiance hyperbolique dans le pouvoir de la divination.

12 La péroraison du discours (510-13) abandonne ainsi logiquement le ton de l'exhortation agressive pour développer a contrario une promesse de récompense : Protésilas se rend compte que la persuasion est plus productive que les menaces. Aussi son raisonnement prend-il ici l'aspect d'un enthymème : « nous t'avons déjà accordé une faveur parce que tu nous es utile comme devin (prémisse majeure)/ or si tu nous amènes Achille tu nous seras encore plus utile (prémisse mineure semi-implicite)/ tu recevras donc une récompense honorifique plus grande encore (conclusion)». On retrouve ici sur un mode plus positif l'allusion aux attributs de la prêtrise et aux honneurs que l'on avait aux v. 505 ; non plus pour désormais en contester la légitimité, mais au contraire pour les confirmer. Le ton est aussi plus apaisé, et les séquences plus amples: Protésilas semble décidé à mettre en sourdine son irritation pour mettre le devin dans de bonnes dispositions. Cette dernière séquence repose sur une problématisation implicite du statut des devins dans l'épopée gréco-latine. Alors que dans l'épopée grecque, les devins sont rarement des combattants ${ }^{48}$, c'est au contraire monnaie courante chez les épiques latins ${ }^{49}$. Protésilas s'appuie donc sur la norme implicite de ces derniers pour présenter comme un régime de faveur découlant de la bienveillance des duces achéens (remisimus) l'exemption de service de Calchas, allant jusqu'à adopter, pour parler au non-combattant qu'est le devin, un vocabulaire "pacifiste" censé lui faire mieux apprécier cette faveur (arma horrenda, saeuos enses, imbelles uittas). Mais ce rappel sert surtout de tremplin à la promesse finale, ou, dans un mouvement de surenchère, Protésilas fait miroiter une reconnaissance morale exceptionnelle à Calchas en cas de contribution décisive à la découverte d'Achille. A l'aune de l'éthique aristocratique dont se réclame le locuteur, la perspective de se voir investi d'un prestige supérieur aux duces (numeroque ducum praestantior omni ${ }^{50}$ ) est censée apparaître aux yeux de son destinataire comme la récompense suprême. Cohérent avec son ethos martial, Protésilas 
métaphorise cette contribution de Calchas (l'apport d'Achille à l'armée) par l'image de la fourniture d'un remplaçant militaire (pro te dependis ${ }^{51}$ ), tout en faisant sentir la disproportion de l'échange (hyperbate emphatique magnum... Achillen encadrant le vers, par contraste avec la brièveté du pronom monosyllabique te désignant Calchas au milieu du vers) : le remplaçant est bien supérieur au remplacé en valeur militaire; ultime touche de condescendance ironique de la part de cet aristocrate guerrier qu'est Protésilas face au prêtre non-combattant.

13 Le discours dessine donc avec vigueur, sous la forme de l'éthopée, le caractère d'un guerrier (un peu trop) sûr de sa valeur et imprégné de mentalité aristocratique, sans trop de respect pour les prêtres, mais néanmoins confiant dans le pouvoir de la divination: un ethos aisément superposable à la figure de Protésilas pour le peu que la tradition légendaire laissait entrevoir de lui (sa hâte malheureuse à courir au combat), moyennant une petite dose d'extrapolation. Dans le contexte du chant I de l'Achilléide, ce personnage joue le rôle d'une incarnation hyperbolique de l'idéal épique, porteparole de valeurs hérö̈ques aux antipodes de celles de Thétis avec qui il forme un violent contraste. La peinture de ce caractère emblématique renforce donc le jeu de contrepoint entre l'univers féminin, pacifique et élégiaque de Scyros qui imprègne la majorité du chant I et l'intermède viriliste, belliqueux et iliadique d'Aulis : deux polarités entre lesquelles se trouve tendu le destin d'Achille.

\section{La portée politique de la scène.}

L'Achilléide ne comporte vraisemblablement pas une visée délibérée d'allégorie politique à la manière de l'Enéide ou de la Pharsale, voire de la Thébaïde ou des Punica, et il n'est pas question ici d'en proposer une « lecture à clés » à base d'éloge ou de critique du pouvoir impérial, voire, suivant une ligne interprétative plus en faveur actuellement, de speculum principis. Toutefois, même dans une épopée "apolitique " dans son principe général, les réalités de la politique romaine peuvent se faire jour ponctuellement et en filigrane pour évoquer de façon vivante et parlante aux yeux du lecteur les enjeux de pouvoir ou de conflit au sein des collectivités que la trame narrative permet d'effleurer. Il me semble que c'est ce qui se passe dans cette scène, où les enjeux de l'attente d'Achille prennent, sous la plume de Stace, une coloration plus politique qu'on ne pourrait s'y attendre, et qui nous amène assez loin, pour le coup, du modèle homérique.

Prenons le début de l'épisode, qui nous dépeint la popularité d'Achille au sein de l'armée (laquelle, rappelons-le, se confond avec le corps civique dans une perspective romaine). Le premier mouvement (467-477) est une ample phrase périodique fondée sur une antithèse dissymétrique: d'abord, une énumération des principaux chefs argiens (au nombre de sept $\mathrm{t}^{52}$ ) et de leurs attributs dans une concessive en cinq parties avec la mise en valeur des noms propres par le jeu des rejets et enjambements (467-72); puis, une principale en quatre temps axée sur la mise en valeur du nom d'Achille répété trois fois ${ }^{53}$ (polyptote, anadiplose) et repris par illum, avec variation verbale (trois verbes à la $3^{\mathrm{e}}$ pers. plur. encadrant un passif personnel) : une structure qui oppose les chefs pris individuellement à Achille soutenu par la troupe. La première partie permet de dérouler un mini-catalogue des chefs que Stace, contrairement à l'habitude épique, dissocie du catalogue des troupes achéennes qui a précédé. Chacun des cinq chefs ou groupes de chefs est caractérisé par un trait emblématique qui renvoie allusivement à l' 
Iliade de façon à créer autour de ces héros une ambiance "hyper-homérique ". Agamemnon et Ménélas (Atridae) sont caractérisés par leur solidarité étroite (pariter, sua bella) et leur empressement belliqueux (capessere), prolongeant l'idée iliadique selon laquelle l'aîné fait pleinement siens les griefs du cadet qui sont à l'origine de la guerre ; une idée abondamment illustrée plus haut (399-401). Le second " couple ", Diomède et Sthénélus, réunit deux héros souvent associés dans l'Iliade ${ }^{54}$ (le second est le cocher du premier) et liés notamment par l'appartenance de leurs pères respectifs (Tydée et Capanée) à l'expédition des Sept contre Thèbes; le motif de l'émulation de la fama paternelle qui est leur caractéristique commune, mis en valeur par l'hypallage (auida uirtute) et par la métaphore inhabituelle à valeur conative (premat ${ }^{55}$ ), recoupe un motif traditionnel: le souci des Epigones d'égaler leurs pères ${ }^{56}$. L'allusion à la jeunesse d'Antiloque dont la valeur n'attend pas le nombre des années renvoie aussi à un motif iliadique (cf. Il. XV, 569). L'ecphrasis de bouclier qui est raccrochée à l'évocation d'Ajax est une allusion à un passage fameux de l'Iliade, VII, 219-220 dont Stace s'inspire de très près (aequantem moenibus orbem ${ }^{57}$ ), rehaussée par une périphrase emphatique (armenti reges $^{58}$ ). Ulysse, qui arrive au climax de cette énumération septénaire pour annoncer son rôle ultérieur dans l'histoire, présente un concentré de mots renvoyant à ses caractéristiques homériques : l'éveil permanent (uigil ${ }^{59}$ ), et l'alliance de compétences guerrières et intellectuelles qui rappelle les préliminaires de la Dolonie (cf. Il., X, 242-47). Bref, pour tous ces chefs, une énumération catalogique de traits homériques, et plus précisément iliadiques, dont l'accumulation emphatique offre un contraste brutal avec leur « dévaluation » face à la popularité du seul Achille.

Celle-ci est développée d'abord dans une principale quaternaire subdivisée en deux groupes binaires. Les deux premières séquences dénotent simplement la popularité du héros, avec variation verbale (ardet/ amant $t^{60}$ ) et en insistant sur le paradoxe d'une popularité in absentia : in absentem... Achillen, nomen Achillis. La triple anaphore avec polyptote du nom propre matérialise ce sentiment populaire à l'instar d'une acclamation par le peuple en armes; une idée qu'évoque aussi, dans le discours de Protésilas, le verbe fremere (499). En outre, cette popularité se fonde, on l'a vu, sur une combinaison de uirtus et d'ascendance divine (479), ce qui rappelle les fondements de la légitimité impériale julio-claudienne ${ }^{61}$. Cet Achille est un peu comme un jeune prince d'une maison impériale, spontanément aimé de la troupe, et dont la légitimité repose essentiellement sur des virtualités, dont l'appartenance à une dynastie prestigieuse est l'un des éléments. Enfin, cette popularité tend vers une adoration quasi religieuse d'Achille en tant que numen (504), en laquelle on peut voir une sorte d'esquisse préfigurative du culte impérial.

17 Cependant la popularité de ce héros à la fois juvénile et déjà jupitérien (cf. v. 488) n'est pas sans susciter, au moins potentiellement, un conflit larvé au sein de la communauté. L'idée procède indirectement du passage de Valérius sur l'abandon d'Hercule, dont nous avons vu qu'il est l'une des sources d'inspiration premières de cet épisode. Mais ce qui chez Valérius relèvait d'une rivalité personnelle entre héros (Méléagre vs Hercule) devient, chez Stace, une source de tension " socio-politique »: le poète souligne bien que d'abord c'est la troupe ( 473 : manus ; 482 : cohortes) qui appelle Achille, et le préfère à ses propres chefs. Cette troupe apparait comme le vecteur d'une sorte de vox populi qu'elle diffuse horizontalement (482: haec Graiae castris iterant traduntque cohortes), et exerce, par sa masse où se mêlent indistinctement les différents contingents, une forme de pression quasi physique sur les délibérations des chefs (492: mixta uallati plebe suorum). Ceux-ci ne semblent réagir que dans un second temps, pour avaliser le choix 
de leurs soldats ( 483 : cedit turba ducum uincique... fatetur). Même si les rois font montre d'un indéniable fair-play dans l'acceptation de la préférence populaire (haud maesta), l'impression est que l'on aurait bel et bien frôlé une sédition au cas (improbable) où ils auraient décidé de se passer d'Achille, comme les Argonautes l'ont fait avec Hercule. Cette tension latente ressort aussi du discours de Protésilas, porte-parole de cette aristocratie guerrière, qui, en reprenant le catalogue des rois infériorisés (500-502), n'est pas sans laisser entrevoir une pointe de jalousie (inspirée du Méléagre de Valérius) face à cette préférence populaire pour l'Eacide. Pourquoi Stace a-t-il jugé intéressant de développer ces suggestions? Certes, la mise en scène d'une forme de tension entre les chefs et la troupe au sein de l'armée a des antécédents iliadiques (cf. Il. II, 200-277), mais la façon statienne d'aborder le problème est radicalement différente de l'approche homérique, assez brutalement pro-aristocratique ${ }^{62}$. De fait, Stace n'écrit pas pour les Grecs de l'époque archaïque, mais pour les Romains de son temps. Ce qu'il met en scène est une société dans laquelle le peuple ${ }^{63}$ nourrit une faveur particulière pour un chef unique et prestigieux de nature semi-divine, par dessus-la tête des différents rois achéens. Ceux-ci sont dès lors ravalés au rang d'une aristocratie censée reconnaître de bon gré sa subordination au leader charismatique. Et de fait, c'est ce qu'ils font, d'assez bonne grâce dans l'ensemble, puisque même Protésilas, qui apparaît comme le représentant le plus coruscant de la mentalité aristocratique, ne conteste pas ouvertement cette situation: ce qui compte par-dessus tout est la réussite de l'entreprise commune, pour laquelle les individus sont prêts à mettre sous le boisseau leurs susceptibilités personnelles, comme il est de règle dans une société bien structurée. C'est cette bonne volonté de l'aristocratie à ratifier le jugement du peuple en armes qui désamorce les tensions potentielles et garantit in fine la cohésion de la communauté. On assiste donc ici à une réinterprétation quelque peu " césarienne » de la société homérique en même temps qu'à une ébauche du culte de la personnalité impériale. Cette démonstration n'est évidemment pas le propos essentiel de l'Achilléide, mais l'on peut dire que Stace a saisi l'occasion que lui donnait la scène d'assemblée de développer un embryon de réflexion politique qu'il savait de nature à trouver un écho dans son lectorat. Voulant faire ressentir l'auctoritas supérieure dont un héros encore " virtuel » peut être investi par une collectivité par ailleurs riche en grands capitaines, il a redessiné la société homérique sous les traits de la cité romaine de son temps, où ce type d'investiture correspond à une réalité désormais bien admise. Au bout du compte, ce n'est pas tant Achille qui représente l'empereur, comme on l'a dit parfois, que l'image de l'empereur qui est récupérée pour mieux faire sentir le charisme préhéroïque d'Achille.

Je voudrais, pour conclure, reprendre la notion de contrepoint, que j'ai mentionnée plus haut, pour caractériser le rapport de ce passage avec la trame principale. Intermède guerrier et néo-homérique au sein de la narration alexandrinisante et à tendance élégiaque de la jeunesse d'Achille, l'épisode d'Aulis impose sa tonalité véhémente et grandiloquente, avec une présence ponctuelle du sublime (la comparaison des v. 484-90) sous la forme d'une « mineure » opposée à la «majeure » de l'épisode scyrien. Cette variété des tonalités, avec un dosage inégal sur le plan quantitatif, est constitutive d'une esthétique de la poikilia qui est un élément essentiel de la personnalité poétique de l'Achilléide ${ }^{64}$ : une épopée qu'on veut trop souvent réduire à sa composante élégiaque et sentimentalisante (tout en prédisant parfois, de façon tout aussi exagérée à mon avis, un basculement intégral dans la fureur et le pathos iliadiques à partir du chant II). C'est pourquoi je suis d'avis que, 
indépendamment des sujets qui devaient être abordés dans la suite de l'Achilléide, (l'amour, ou la guerre, ou les deux à la fois) cette technique du contrepoint devait rester une caractéristique constante de l'œuvre, avec une tonalité dominante détendue et plaisante, et quelques " poussées de tension » pathétiques et sublimes, d'autant plus frappantes que parcimonieusement distribuées d'une part, et surtout, nettement circonscrites et de courte durée d'autre part. En effet, une autre caractéristique de l' Achilléide, au sein même de cet épisode martial d'Aulis, est le parti pris de dédramatisation ${ }^{65}$. Contrairement à l'Iliade, les passions et les tensions n'y arrivent jamais à un point de rupture : les chefs achéens cèdent de bon gré à la préférence de la troupe, et Protésilas finit son discours d'exhortation à Calchas sur un ton plus conciliant qu'il ne l'avait commencé. Epopée « éthique » est l'Achilléide, épopée éthique elle reste, et nous retombons bien, in fine, dans la sphère des adfectus mites atque compositi caractéristiques de cet univers poétique, comme le dit Quintilien ${ }^{66}$ (Inst. Or., VI, 2, 9). Stace met en place dans cette scène une situation virtuellement porteuse de tensions et renvoyant allusivement à des moments d'affrontement parfois vif chez ses modèles épiques (la scène d'assemblée d'Il. II, le débat entre Méléagre et Télamon chez Val. Fl. III, la scène entre Capanée et Amphiaraos dans Theb. III) pour mieux en désarmorcer les potentialités conflictuelles grâce à la bonne volonté de ses personnages. De même, Lycomède se calme assez vite après s'être rendu compte qu'il avait été dupé (I, 912-18), et le regret d'Achille après sa séparation d'avec Déidamie est de courte durée (II, 27-32). Un autre exemple de " dégonflage » d'une situation de crise mise en place notamment par un jeu d'allusivité intertextuelle est la scène initiale (20-94), où la démarche de Thétis auprès de Neptune pour engloutir la nef de Pâris semble annoncer une tempête épique analogue à celle suscitée par Junon contre Enée au chant I de l'Enéide, avant que les intentions agressives de la Néréide ne viennent butter de façon déceptive contre l'anti-interventionnisme du dieu des mers (cf. 95-98). A côté de l'esthétique du contrepoint dont j'ai parlé, ces exemples permettent aussi de parler d'une esthétique du decrescendo, qui est une autre originalité de l'Achilléide dans le paysage épique latin.

\section{BIBLIOGRAPHIE}

ARICÒ 1986 : G. Aricò, « l'Achilleide di Stazio : tradizione letteraria e invenzione narrativa », ANRW II. 32. 5, 1986, p. 2925-2964.

BARCHIESI 1996 : A. Barchiesi, « La guerra di Troia non avrà luogo : il proemio dell'Achilleide di Stazio ", AION (filol) 18, 1996, p. 45-62.

BESSONE 2016 : F. Bessone, « The Hero' Extended Family : Familial and Narrative Tensions in Statius' Achilleid », in Family in Flavian Epic, N. Manioti ed., Leiden-Boston, 2016, p. 174-208.

BITTO 2016 : G. Bitto, Vergimus in senium : Statius' Achilleis als Alterswerk, Göttingen, 2016.

DELARUE 2000 : F. Delarue, Stace poète épique. Originalité et cohérence, Louvain-Paris 2000. 
DILKE 1954 : O. A. W. Dilke, Statius. Achilleid. Edited with Introduction and Commentary, Cambridge 1954.

FANTHAM 1999 : E. Fantham, « Chironis Exemplum : On Teachers and Surrogate Fathers in Achilleid and Silvae », Hermathena 67, 1999, p. 59-70.

FEENEY 2004 : « Tenui... latens discrimine : Spotting the Differencies in Statius' Achilleid », MD 52, 2004, p. 85-105.

HARDIE 2013 ; Ph. Hardie, « Flavian Epic and the Sublime », in Flavian Epic Interactions, G. Manuwald \& A. voigt eds, Berlin, 2013, p. 125-138.

HESLIN 2005 : P. J. Heslin, The Transvestite Achilles. Genre and Gender in the Achilleid, Cambridge, 2005.

MÉHEUST 1971 : Stace. Achilléide. Texte établi et traduit par J. Méheust, Paris, 1971.

MOUL 2012 : V. Moul, «Quo rapis ? Tone and Allusion at Aulis in Statius'Achilleid », CQ 62, 2012, p. 286-300.

NUZZO 2012 : G. Nuzzo, Publio Papinio Stazio. Achilleide, Palerme 2012.

PARKES 2008 : "The Return of the Seven : Allusion to the Thebaid in Statius' Achilleid », AJPh 129, 2008, p. 381-402.

PARKES 2014 : «The Epics of Statius and Valerius Flaccus' Argonautica », in Brill's Companion to Valerius Flaccus, M. Heerink \& G. Manuwald eds, Leiden-Boston, 2014, p. 326-339.

RIPOLL-SOUBIRAN 2008 : F. Ripoll et J. Soubiran, Stace. Achilléide. Louvain-Paris-Dudley, Ma, 2008.

RIPOLL 2010 : F. Ripoll, « La guerre de Troie dans l'Achilléide de Stace », Rursus-Spicae [En ligne], 5 | 2010, mis en ligne le 11 mars 2010. URL : http://journals.openedition.org/rursus/413.

RIPOLL 2014 : F. Ripoll, « Mémoire de Valérius Flaccus dans l'Achilléide de Stace », REA 116, 2014, p. 83-103.

ROSATI 1992 : G. Rosati, « L'Achilleide di Stazio, un'epica dell'ambiguità », Maia 44, 1992, p. 233-266.

SÉCHAN 1953 ; L. Séchan, « La légende de Protésilas », BAGB Lettres d'humanité 12, 1953, p. 3-27.

\section{NOTES}

1. Pour une approche générale de l'épisode, voir principalement Bitto 2016, p. 266-274.

2. Le texte suivi ici est tiré de Ripoll-Soubiran 2008. La traduction est personnelle.

3. Par exemple, la désignation d'Ulysse et Diomède comme ambassadeurs (536-59) est une «préfiguration a posteriori » de la Dolonie homérique (Il. X, 218-53); de même la réception d'Ulysse chez Lycomède (726-840) annonce par certains traits celle d'Ulysse chez Alcinous aux chants VII-VIII de l'Odyssée.

4. Sur la présence de Valérius Flaccus dans l'Achilléide en général, voir Parkes 2014 et Ripoll 2014.

5. Un épisode que le poète flavien avait considérablement amplifié par rapport à Apollonios (I, 1274-1344).

6. Variation sur le motif aliquem solum in certamina poscere, fréquent dans l'épopée, notamment (mais pas exclusivement) dans des contextes de défi : cf. Aen., IX, 439 ; X, 662 ; XII, 467 ; Theb, XI, 245 ; Ach., I, 922. 
7. Le modèle, en amont de Valérius, est le discours de Turnus chez Virgile, Aen., XI, 428-440, mais Stace est plus près de Valérius que de Virgile du fait de la présence latente, dans les deux cas, d'une figure de héros absent et populaire en antithèse à cette revue de chefs.

8. C'est en fait c'est l'ensemble des v 500-501 de Stace qui été influencé par Valérius : Calydonius heros/ et magno genitus Telamone.

9. Sur l'influence des Skyrioi dans l'Achilléide, voir notamment Aricò 1986.

10. Ulysse parlera plus loin à ce propos (II, 33) d'oracles dont il n'aura pas été question auparavant, mais qui renvoient à la tradition issue des Chants Cypriens (cf. aussi, en plus vague II, 57) ; ce qui montre bien que la cohérence narrative dans le détail est un souci très secondaire du poète : en bon élève des Alexandrins, il n'hésite pas à faire ressurgir à un autre moment du récit (pour des raisons spécifiques à ce moment-là) une version du mythe qu'il a écartée dans la narration principale (pour des raisons elles aussi spécifiques).

11. Voir note ad loc de Ripoll-Soubiran 2008.

12. Adstrictos didici reptare per amnes.

13. Cf. le discours du Scythe Gésandre chez Valérius, VI, 325 et 335-37.

14. Cf. aussi Val. Fl., VI, 325 : altricemque niuem.

15. Cf. Ov., Met., VII, 264.

16. Sur le rôle formateur de Chiron dans l'Achilléide et notamment la tension entre rudesse militaire et tendresse quasi maternelle, voir Fantham 1999 et Bessone 2016 (spécialement p. 177-84).

17. Cruda rudimenta est une variation sur la junctura virgilienne à coloration militaire dura rudimenta (Aen. XI, 156), sans doute amenée à l'esprit du poète par la médiation de Valérius III, 600 (grata rudimenta), dans un passage dont nous avons plus haut que Stace s'en était inspiré sur un plan plus général. La variatio : cruda au lieu de dura est peut-être une allusion oblique à la tradition sur l'alimentation d'Achille à base d'entrailles d'animaux vivants (cf. Ripoll-Soubiran, p. 292 pour les réf. et la bibliogr.).

18. Première attestation de ce mot après Silv., III, 44 au sens de « lignage ».

19. Voir Ripoll-Soubiran 2008, p. 151-52 à propos de I, 2 (patrio caelo).

20. Voir aussi v. 134 (avec la note ad loc de Ripoll-Soubiran 2008, p. 174) et 269. Cette version a fini par supplanter la version traditionnelle de l'immunisation par le feu.

21. Datif de destination ici.

22. Probable allusion oblique à l'armure forgée pour Achille par Héphaïstos à la demande de Thétis (Il., XVIII, 458-61).

23. Cf. Aen., IX, 433.

24. Souvenir de la version selon laquelle Thétis effectuait les rites d'immunisation d'Achille (en l'occurrence par le feu) à l'insu de Pélée (cf. Ap. Rhod., IV, 869-75).

25. Cf. Ripoll-Soubiran, p. 14-15.

26. Sur cet effet d'entrecroisement, voir Ripoll 2010.

27. Voir Ripoll 2014, p. 90-92.

28. Ces trois dieux figuraient chez Lucain, VII, 145-50 (cf. en amont Hor., Od., III, 55-64 et Etna, 61-63). L'image imposante de Mars dressé avec sa lance prolonge des expressions virgiliennes analogues avec l'épée ou le bouclier (Aen., IX, 749 ; XII, 720 ; XI, 284). L'image des serpents dressés de l'égide de Pallas continue cette idée de verticalité (surgeret/ tolleret). Quant à l'image d'Apollon bandant son arc contre les ennemis (qui apporte une variation avec l'idée de courbure), elle rappelle l'ecphrasis de la bataille d'Actium, Aen., VIII, 704, contaminée pour l'expression avec Ov., Am., III, 3, 29.

29. On est donc dans une comparaison à correspondances multiples, comme les aime Stace : Jupiter = Achille ; les chefs grecs = les dieux olympiens (sur un plan général) ; et implicitement, les Géants = les Troyens. 
30. Valérius lui-même s'est sans doute souvenu, pour le motif de la peur des dieux, d'Ovide, Met., V, 322 (je remercie G. Rosati pour cette suggestion). En amont, on peut citer aussi Horace, Od., III, 4, 49 et Etna, 54 (peur de Jupiter menacé par l'attaque des Géants). Stace infléchit la suggestion de la peur collective en la reportant sur les autres dieux, pour mieux détacher la figure souveraine de Jupiter.

31. Ce précédent est une puissante raison de maintenir au v. 484 la leçon pallentes, que certains (Willamowitz) suggèrent de corriger en Pallenes (nom de la presqu'île où se situe la Gigantomachie) en pensant notamment à Luc., VII, 150 et VF II, 17 où figure ce nom. Mais il n'est pas impossible que l'allusion à Pallène dans ce passage de Valérius et/ ou de Lucain ait donné l'idée à Stace, par paronomase, de traduire la peur des dieux par le participe pallentes, avec peutêtre l'esquisse d'un jeu de mots (Soubiran).

32. Sur l'association entre Jupiter et Achille, et la tension liée à ce motif dès le prologue (1-2), voir Barchiesi 1996.

33. Sur le Sublime dans l'épopée flavienne, voir en dernier lieu Hardie 2013.

34. Cf. les paroles de Chiron en I, 147-152. Voir à ce propos Bessone 2016, p. 183 et Heslin 1005, p. 296.

35. Sur l'Achilléide comme épopée " éthique " par opposition à l'épopée "pathétique ", voir Delarue 2000, p. 191-231.

36. Notamment, pour le v. 493, cf. Aen II, 122 : Hic Ithacus uatem magno Calchanta tumultu/protrahit in medios. Cela rejoint, en amont, le motif traditionnel du devin qui doit subir une contrainte violente pour délivrer sa prophétie (cf. la figure de Protée et le Silène de la $6^{\mathrm{e}} \mathrm{Buc}$.).

37. Cf. notamment : heu pudeat (Theb. III, 609) et pudet heu (Ach. I, 503). Pour un parallèle détaillé, voir Bitto 2016, p. 269-74. Sur ce parallélisme et sur les réminiscences de la Thébaïde dans l' Achilléide de façon plus générale, voir Parkes 2008.

38. Cf. aussi, plus ponctuellement, Agamemnon face à Chrysès, Il., I, 28.

39. Voir Séchan 1953.

40. Cf. par ex. l'allusion (involontaire) d'Ulysse au début de l'Odyssée (Od., I, 3) en I, 785-87.

41. Mouvements analogues chez Virg., Aen. IV, 267 ; Ov. Her., I, 41 ; Sil. Pun., XII, 69.

42. Pour le choix de la leçon quianam... recludes et son interprétation, voir Ripoll-Soubiran, 2008 p.

222. A l'appui de l'interprétation de J. Soubiran, j'ajouterais que le mouvement des vv. 497-498 n'est pas un vrai questionnement, mais un mouvement rhétorique traduisant l'exaspération de Protésilas. Outre le fait que la leçon quaenam («quels secrets révèleras-tu?») ressemble à une correction banalisante, cela introduit une dissymétrie en posant, pour le coup, une vraie question, d'où un effet d'anticlimax inadéquat avec le mouvement de l'ensemble (aggravé d'une platitude). De même, l'interprétation de quianam recludis (en corrigeant, comme le suggèrent certains éditeurs, recludes en recludis et en prenant ce verbe au sens, plus rare, de « cacher ») par «pourquoi caches-tu » aboutit aussi à une platitude. Je pense en fait que la deuxième question redouble la première en franchissant un degré supplémentaire dans l'exaspération de Protésilas ; le sens est donc: «pour quel meilleur motif (sous-entendu : que celui-ci) révèras-tu... » (iustius est en fait commun aux deux questions; c'est l'interprétation de Brinkgreve, récusée sans argument valable par Dilke mais reprise par Méheust et Soubiran). D'autre part, le « aut » suggère que la deuxième question va dans le même sens que la première avec qui elle constitue une redondance rhétorique doublée d'un effet de surenchère.

43. Pour le choix de arrepta au lieu de abrepta, voir Ripoll-Soubiran 2008, p. 222-23.

44. Adulation des soldats de Scaeva pour leur chef : ac uelut... numen/ et uiuam magnae speciem Virtutis adorant.

45. Notons au passage que les deux hypothèses envisagées par Protésilas renvoient aux deux versions concurrentes sur l'enfance du héros : d'une part, celle d'Homère (Il., XVIII, 57-60 ; XI, 485-95), selon laquelle Achille a été élevé dans le palais de son père, et d'autre part, la vulgate issue des Chants Cypriens et représentée notamment par Apollonios de Rhodes (IV, 852-81), selon 
laquelle il a été confié entièrement à la garde de Chiron; c'est la seconde que Stace choisit de suivre dans la narration principale, mais il fait ici une allusion sur le mode alexandrin à celle qu'il a récusée, pour suggérer que, dans leur quête d'Achille, les Achéens ont exploré toutes les possibilités attestées par la tradition littéraire.

46. Pour cette expression, à prendre au sens propre (cf. Ripoll-Soubiran 2008, p. 223 ; contra Dilke 1954, p. 119), voir Theb., X, 605 (contexte de capnomancie comme ici).

47. Epithète d'Apollon (cf. Ov., Am., III, 389), ici au sens de "prophétique » par extension métonymique.

48. Une exception : Il. II, 858-61; à quoi il faut ajouter le cas particulier d'Amphiaraos.

49. Pour les références, voir Ripoll-Soubiran 2008, p. 223.

50. Ov., Met., III, 54.

51. Pour le choix de dependis (au lieu de portendis ou deprendis), voir Méheust 1971, p. 29 (et Dilke 1954, p. 119).

52. Cf. Nuzzo 2012, p. 104, qui insiste sur la valeur symbolique de ce chiffre (que l'on retrouve au v. 470) et parle de «Sept contre Troie».

53. Cette triple conclamatio rappelle Theb., XII, 805-807 (Arcada = Parthénopée), et, en amont, Virg., Buc., VI, 53-44 et Georg., IV, 525-27. Voir à ce propos Feeney 2004, p. 87-88.

54. II, 563-64 ; IV, 365-67 ; V, 106-10 ; 239-42 ; 318-20 ; 835-37 ; VIII, 114-15 ; XXIII, 510-13.

55. Cf. Silv., I, 2, 116.

56. Cf. Il. IV, 365-400 pour Diomède.

57. Avec une clausule ovidienne; cf. Pont., II, 1, 12 (moenibus orbem).

58. Cf. Theb., XI, 28.

59. Cf. notamment Od. XIX, 336-42.

60. Sur l'emploi du lexique érotico-élégiaque dans ce passage (ardent, amant...), voir Moul 2012, p. 293.

61. Il est vrai que Stace écrit sous les Flaviens, qui n'avaient pas les mêmes prétentions : cf. Suét., Vesp., 12; mais l'anecdote suétonienne atteste précisément que le modèle julio-claudien de légitimation par l'ascendance divine restait prégnant malgré la position personnelle de Vespasien.

62. Cf. Il., II, 243-77.

63. Cf. 491 : plebe.

64. Au reste, les Métamorphoses d'Ovide, considérées à juste titre comme un modèle majeur de l' Achilléide, font aussi cohabiter des scènes pittoresques et alexandrines avec des tableaux grandioses comme le déluge ou l'équipée de Phaéton.

65. Sur la dédramatisation dans l'Achilléide, voir Ripoll-Soubiran 2008, p. 55-60 et Rosati 1992, p. 245.

66. Voir Delarue 2000, p. 210-14.

\section{RÉSUMÉS}

La section de l'Achilléide de Stace qui prend place entre le rassemblement des Grecs à Aulis et la prophétie de Calchas (467-513) est une scène de transition qui a peu retenu l'attention des commentateurs. Elle est cependant intéressante à étudier, à côté des "morceaux de bravoure » centrés sur le travestissement d'Achille, pour saisir le projet du poète dans sa globalité. Elle 
illustre en effet une technique du contrepoint dans cette épopée qui orchestre, sur le mode mineur et à échelle réduite, une esthétique néo-iliadique à tendance sublime en contraste avec l'ambiance élégiaque et alexandrine de la narration principale. Complémentairement, elle met aussi en œuvre une stratégie de dédramatisation et de decrescendo en jouant sur l'allusion à des intertextes puissamment conflictuels pour les désamorcer au profit d'une ambiance "éthique » fondée sur la modération des affects. Elle présente en outre la particularité de mobiliser des références indirectes à des modèles politiques romains pour nourrir l'évocation de la société homérique et la rapprocher de l'époque contemporaine. Une étude littéraire d'ensemble de cette scène mettra en lumière ces divers aspects.

INDEX

Mots-clés : Stace, Achilléide, Achille, Protésilas, Calchas, intertextualité, prophétie, sublime, gigantomachie, Iliade, Valérius Flaccus. 